\section{The powerful advantages of extracellular electrical recording}

\section{Jan D. Huizinga}

Extracellular electrical recordings have been, and continue to be, extremely helpful in understanding the rhythmic pacemaker activities in the gut. In a recent Perspectives article (Problems with extracellular recording of electrical activity in gastrointestinal muscle. Nat. Rev. Gastroenterol. Hepatol. 13, 731-741; 2016) ${ }^{1}$, attempts have been made to question this technique, which is unfortunate owing to the potential discreditation of seminal previous and current studies. The main issue reported by Sanders et al. ${ }^{1}$ is that movements can cause artefacts in the electrical recordings, but anyone who works with extracellular electrodes is aware of this issue and how potential pitfalls can easily be avoided ${ }^{2}$.

Alvarez $^{3}$ was the first to record electrical activity of the gut in 1922 and he already understood the issue of movement contamination: "the most remarkable thing about these action currents is that they are produced constantly in stomachs and intestines which, so far as the eye can detect, are absolutely motionless". Furthermore, Alvarez noted that the electrical activity was similar whether contractions occurred or not. Extracellular recording is a very powerful technique as it can be

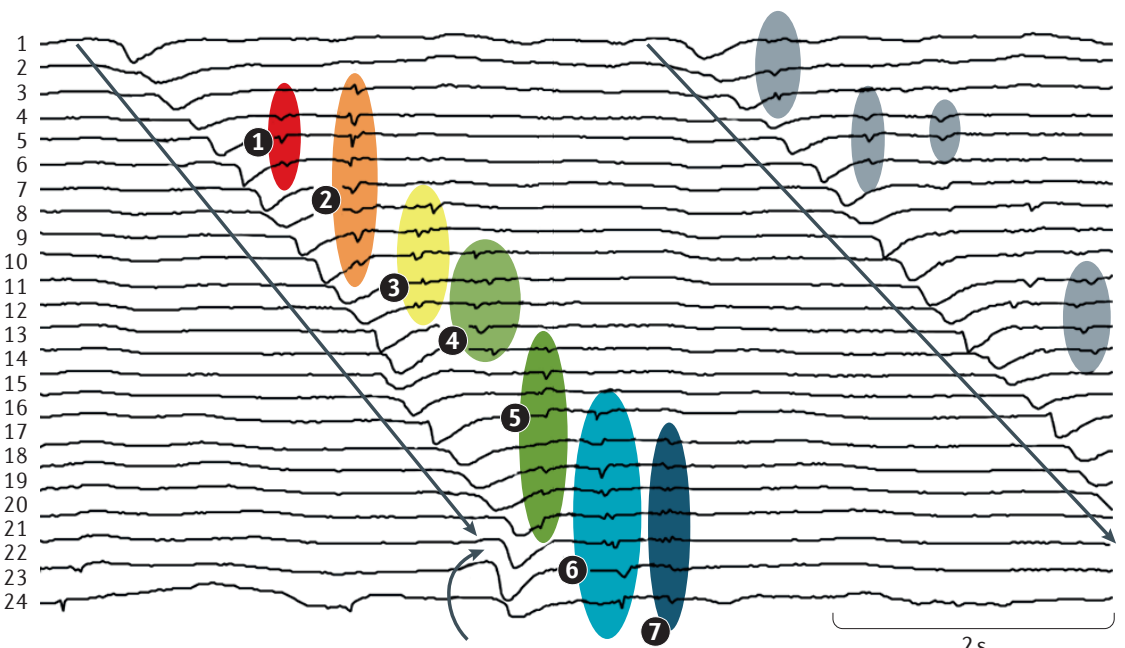

b

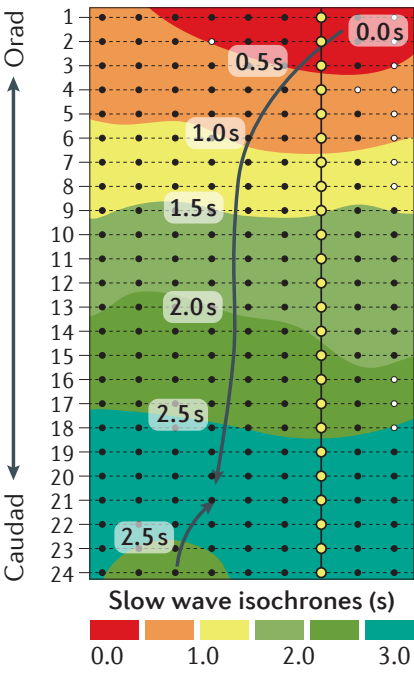

c

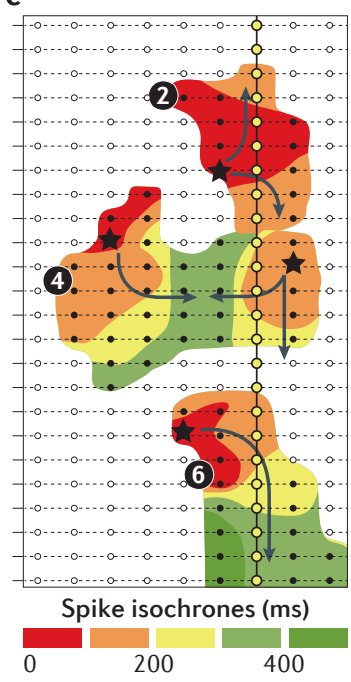

d

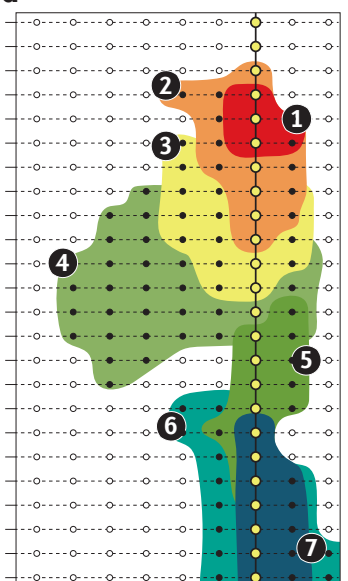

Spike number

$\begin{array}{lllllll}1 & 2 & 3 & 4 & 5 & 6 & 7\end{array}$

performed in vivo or ex vivo in whole organs; hence, in complete or near complete physiological conditions. They can also be recorded even when powerful contractions occur, a feat no other technique to measure electrical activity can accomplish; hence, an advantage of the technique, not a problem.

In Figure 3 from their article, Sanders et al. ${ }^{1}$ show an example in which electrical activity disappears when contraction stops. However, those familiar with this technique know that this situation can happen with a poor electrode position. Lammers and co-workers have contributed enormously to our understanding of the network properties of the interstitial cells of Cajal by recording, amongst others, slow-wave propagation in the small intestine of a cat simultaneously with 240 extracellular electrodes ${ }^{4}$. As the purpose was to understand fundamental properties of pacemaker activity, the experiments were done in the absence of contractile activity. Only this technique could have proven that slow-wave activity easily traverses from the proximal end of the intestine all the way to the distal intestine ${ }^{4}$. Only this technique could have demonstrated the paucity of pacemaking activity at the pyloric sphincter in rats and mice, which is needed to insulate the different activities and functions of gastric and duodenal pacemaking 5 .

Figure 1 | Extracellular recording from a cat small intestine showing propagating slow waves and associated smooth muscle action potentials (spikes) recorded using a 240-electrode array. a | Electrogram of typical spike activities after a single slow wave, displayed using the signals from 24 of a 240-electrode array. The positions of the 24 electrodes are shown by yellow circles in $\mathbf{b}-\mathbf{d}$ corresponding to numbers shown on the left of $\mathbf{a}$ and $\mathbf{b}$. Propagating spikes are shown within coloured ovals marked with black circled numbers $1-7$. The grey ovals show spikes after the second slow wave. b|Propagation of the first slow wave shown in a. Black circles indicate electrodes where activity was recorded, white circles indicate no activity. c| Activation sequence of spike propagation in spike patches 2, 4, and 6. A spike patch is an area activated by a single smooth muscle action potential. Isochrones in spike patches were drawn every $100 \mathrm{~ms}$. Spikes originated at circumscribed locations indicated by black stars, and propagated in several directions for 100-400 ms before terminating abruptly. d | Composite spike map displays boundaries of all seven spike patches, showing considerable variations in size, location and overlap between patches. Modified with permission obtained from the American Physiological Society (C) Lammers, W. J. Am. J. Physiol. Gastrointest. Liver Physiol. 278, G297-G307 (2000). 


\section{CORRESPONDENCE}

Extracellular electrodes are sensitive enough to distinguish slow waves from smooth muscle action potentials, and detailed knowledge has emerged from this technique regarding the behaviour of patches of action potentials superimposed on the slow waves ${ }^{6}$ (FIG. 1). Another example is our understanding of the waxing and waning electrical slow-wave activity that accompanies the classic segmentation motor pattern of the small intestine ${ }^{7}$. As this activity is accompanied by vigorous contractile activity, a flexible extracellular electrode is the perfect way to record accompanying electrical activity. After we performed this technique in rats, we confirmed the waxing and waning pattern with intracellular electrodes in mice and showed that the patterns of intracellular and extracellular activity were the same ${ }^{7}$.

Detailed slow-wave conduction abnormalities were recorded recently in patients with gastroparesis - an amazing accomplishment that will be of critical importance in elucidating one aspect of the pathophysiology of this disease $e^{8}$. The future of science lies in the elucidation of mechanisms by a variety of methods and extracellular recording needs to be embraced as a powerful technique, and sometimes the only one suitable technique.

Jan D. Huizinga is at McMaster University, Farncombe Family Digestive Health Research Institute, 1200 Main Street West, Hamilton, Ontario L8N 3Z5, Canada. huizinga@mcmaster.ca

doi: $10.1038 /$ nrgastro.2017.16

Published online 30 Mar 2017

1. Sanders, K. M., Ward, S. M. \& Hennig, G. W. Problems with extracellular recording of electrical activity in gastrointestinal muscle. Nat. Rev. Gastroenterol. Hepatol. 13, 731-741 (2016).

2. Angeli, T. R. et al. The bioelectrical basis and validity of gastrointestinal extracellular slow wave recordings. J. Physiol. 591, 4567-4579 (2013).

3. Alvarez, W. C. Action currents in stomach and intestine. Am. J. Physiol. 58, 476-493 (1922).
4. Lammers, W. J. \& Stephen, B. Origin and propagation of individual slow waves along the intact feline small intestine. Exp. Physiol. 93, 334-346 (2008).

5. Wang, X. Y., Lammers, W. J., Bercik, P. ¿ Huizinga, J. D. Lack of pyloric interstitial cells of Cajal explains distinct peristaltic motor patterns in stomach and small intestine. Am. J. Physiol. Gastrointest. Liver Physiol. 289, G539-G549 (2005)

6. Lammers, W. J., Slack, J. R., Stephen, B. \& Pozzan, O. The spatial behaviour of spike patches in the feline gastroduodenal junction in vitro. Neurogastroenterol. Motil. 12, 467-473 (2000)

7. Huizinga, J. D. et al. The origin of segmentation motor activity in the intestine. Nat. Commun. 5, 3326 (2014).

8. O'Grady, G. et al. Abnormal initiation and conduction of slow-wave activity in gastroparesis, defined by highresolution electrical mapping. Gastroenterology 143 , 589-598.e1 (2012).

9. Lammers, W. J. Propagation of individual spikes as "patches" of activation in isolated feline duodenum Am. J. Physiol. Gastrointest. Liver Physiol. 278, G297-G307 (2000).

Acknowledgements

I gratefully acknowledge that Professor Wim Lammers provided the original version of Figure 1, provided minor adjustments and gave permission to use the figure.

Competing interests statement

The author declares no competing interests. 